\title{
A JoInt Timing OfFSET AND CHANNEL ESTIMATION USING FRACTIONAL FOURIER TRANSFORM AND CAZAC SEQUENCES
}

\author{
Cherif Rezgui and Khaled Grayaa \\ Communication Systems Lab, National School of Engineers of Tunis (ENIT), Tunisia
}

\begin{abstract}
This paper deals with symbol timing offset and channel estimation in OFDM (orthogonal frequency division multiplexing) system in fast varying channel. Symbol timing offset (STO) estimation is a major task in OFDM. Most of existing methods for estimating STO used cyclic prefix or training sequences. In this paper, we consider a new system for STO estimation using constant amplitude zero autocorrelation (CAZAC) sequences as pilot sequences in conjunction with fractional Fourier transform (FRFT). After STO estimation is done, timing compensation is made. Thereafter, channel is estimated to well recover the original transmitted signal. This method gives good results in terms of MSE in comparison with other known techniques, it estimated well the channel and it is important for fast varying channel. MATLAB Monte-Carlo simulations are used to evaluate the performance of the proposed estimator.
\end{abstract}

\section{KEYWORDS}

OFDM, STO, FRFT, CAZAC sequences, MSE, LS, MMSE, SNR

\section{INTRODUCTION}

Multicarrier modulations are increasingly used in various telecommunication systems such as in Digital Audio Broadcasting (DAB), Digital Video Broadcasting Terrestrial (DVBT), digital broadband communications, Long Term Evolution (LTE), WiMAX,... The principle of OFDM is to divide, on a wide number of subcarriers, the digital signal that is to be transmitted. In wireless systems, transmitted data reaches to receivers after transit through a radio channel. For conventional coherent receivers, the effect of channel on transmitted signal must be estimated to recover transmitted data.

In so far as the receiver estimates exactly how the channel changes the transmitted signal, it can recover sent data. The signal is spread in frequency, time, and angle; therefore, the received signal suffers from the impact of these spreads on transmitted signal.

IFFT and FFT are basic functions necessary for modulation and demodulation in the OFDM systems. To take the N Point FFT at the receiver, it is necessary that exact samples of transmitted information for the OFDM symbol period. In other terms, symbol synchronization must be carried out in order to detect the starting point of each OFDM symbol (after removing CP), which facilitates obtaining the exact samples. Therefore, STO must be estimated by the receiver.

Estimated STO is then compensated witch called timing synchronization. Timing synchronization is one of the major challenges of OFDM system. Imperfect synchronization destroys the orthogonality of sub-carriers and deteriorates the performance of OFDM system. Timing synchronization consists of symbol timing offset estimation and correction. Many techniques are 
used literature to compensate this STO, using cyclic prefix or training sequences as preamble. After synchronization is done, the OFDM channel is estimated in order to best recover the transmitted symbols. OFDM channel estimator uses different techniques such as LS with different type of interpolation and MMSE with many variant. In this paper, we propose a receiver with timing synchronization and channel estimation based on fractional Fourier transform and Constant Amplitude Zero Auto-Correlation (CAZAC) as pilot sequences.

The remainder of this paper is organized as follows. In section 2, we introduce OFDM signal and the effect of STO. In section 3, we introduce some techniques used in the literature estimating STO. Section 4 studies the most used channel estimation techniques and gives comparison between them. Then, we present Fractional Fourier Transform in section 5. Thereafter, Section 6 shows the proposed method. Finally, Section 7 shows the performance of this technique in terms of MSE. Conclusion is given in the last section.

\section{OFDM AND EFFECT OF STO}

OFDM is a multicarrier modulation technique which divides the transmission band into $\mathrm{N}$ several sub-channels that are transmitted in parallel. Compared to the single-carrier modulation,the OFDM symbols have a relatively long duration, but a narrow bandwidth. Accordingly, the OFDM is more robust to the multipath channel dispersion, ensuring the frequency selectivity of the mobile radio channel resistance. The OFDM symbol is defined as $\left\{X_{n} ; n=0 ; 1 ; \ldots ; N-1\right\}$. The OFDM signal is as follows:

$$
x_{l}(t)=\frac{1}{\sqrt{N}} \sum_{k=0}^{N-1} X_{l}(k) e^{j 2 \pi \Delta f k t} ; 0 \leq t<N T
$$

Where $\mathrm{j}=\sqrt{ }-1, \Delta \mathrm{f}$ is spacing between, 1 the $1^{\text {th }}$ OFDM symbol and NT is data block period. The subcarriers in OFDM system are supposed to be orthogonal to each other. In order to correctly demodulate an OFDM symbol at the receiver using $\mathrm{N}$ point DFT, it is very important to take exact samples of transmitted OFDM symbol. The correct starting point of DFT window is needed to preserve the orthogonality among the sub-carriers. OFDM systems present many advantages in comparison with single carrier system however all these advantages can be relevant only when the orthogonality among sub-carriers is kept. If one DFT window takes sample of two different OFDM symbol generating ICI and ISI. Table 1 shows the effect of timing offset in the received signal in time and frequency domain. Channel effects and noise are neglected for simplicity. The presence of STO in the time domain causes phase offset of $2 \pi \mathrm{kd} / \mathrm{N}$ in the frequency domain. This phase offset is proportional to the subcarrier index $\mathrm{k}$ as well as the STO $\delta$, Table 1 .

Table 1: The effect of STO on the received signal, [1]

\begin{tabular}{|l|l|l|}
\hline & \multicolumn{1}{|c|}{ Received signal } & Effect of STO $\delta$ on the received signal \\
\hline Time-domain signal & $\mathrm{y}(\mathrm{n})$ & $\mathrm{x}(\mathrm{n}+\delta)$ \\
\hline Frequency-domain signal & $\mathrm{Y}(\mathrm{k})$ & $\mathrm{e}^{\mathrm{j} 2 \pi \mathrm{k} \delta} / \mathrm{N} \mathrm{X}(\mathrm{k})$ \\
\hline
\end{tabular}

Four possible cases may occur, Figure 1. 
Case I: No timing error is presented in this case (i.e. timing offset $\delta=0$ ). That is the case that estimated starting point of the OFDM symbol coincides with the exact time, preserving the orthogonality among sub-carriers, so the OFDM symbol can be perfectly left without any type of interference.

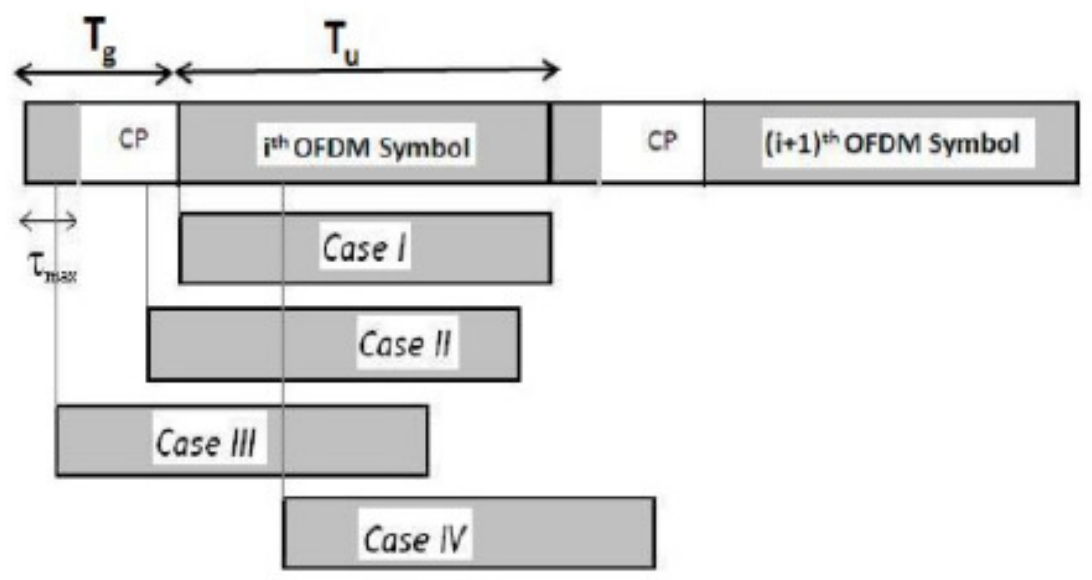

Figure 1: Four different cases symbol starting point subject to STO.

Case II: That is the case that the estimated starting point of the OFDM symbol before the exact point, but after the end of the (displaced) channel response of the precedent OFDM symbol. In that case the $\mathrm{i}^{\text {th }}$ symbol not overlapped with the preceding $(\mathrm{i}-1){ }^{\text {th }}$ OFDM symbol, that is, without incurring ISI by the preceding symbol in such cases. The received signal after passing through FFT block time- domain received samples $\left\{x_{l}(n+\delta)\right\}_{n=0}^{N-1}$, given as:

$$
\begin{aligned}
Y_{l}(k) & =\frac{1}{N} \sum_{n=0}^{N-1} x_{l}(n+\delta) e^{-j 2 \pi n k / N} \\
& =\frac{1}{N} \sum_{n=0}^{N-1}\left\{\sum_{p=0}^{N-1} X_{l}(p) e^{j 2 \pi(n+\delta) p / N}\right\} e^{-j 2 \pi n k / N} \\
& =X_{l}(k) e^{j 2 \pi k \delta / N}
\end{aligned}
$$

The term in equation 2 indicates that the orthogonality between the subcarriers of the frequency components can be fully preserved. However, there is a phase offset. This phase offset leads to rotation of signal constellation around the origin.

Case III: This case occurs when starting point of the OFDM symbol is estimated exist prior to the end of the (displaced) channel response of the previous OFDM symbol and thus the symbol synchronization is too soon to avoid the ISI. In that case the orthogonality between the components of subcarrier is destroyed by the ISI (the previous symbol) and furthermore, ICI occurs.

Case IV: That is the case if the starting point of the OFDM symbol is estimated just after the exact time. In this case, the samples for current FFT operation interval is consists of a part of the current OFDM symbol $\mathrm{x}_{1}(\mathrm{n})$ and a part of the following OFDM symbol $\mathrm{x}_{1+1}(\mathrm{n})$. 
International Journal of Computer Networks \& Communications (IJCNC) Vol.8, No.2, March 2016

As illustrated, an STO can lead not only to phase distortion, but also ISI in OFDM systems. Therefore, timing synchronization must be done at the receiver. Synchronization task is composed by STO estimation and compensation. Generally, the STO estimation can be used either in the time or frequency domain.

\section{RELATED WORK FOR ESTIMATING STO}

Many techniques in the literature are implemented using whether cyclic prefix or training sequence. As an example of estimating STO using cyclic prefix is done by Tourtier, P.J., Monnier, R., and Lopez, P. [2].

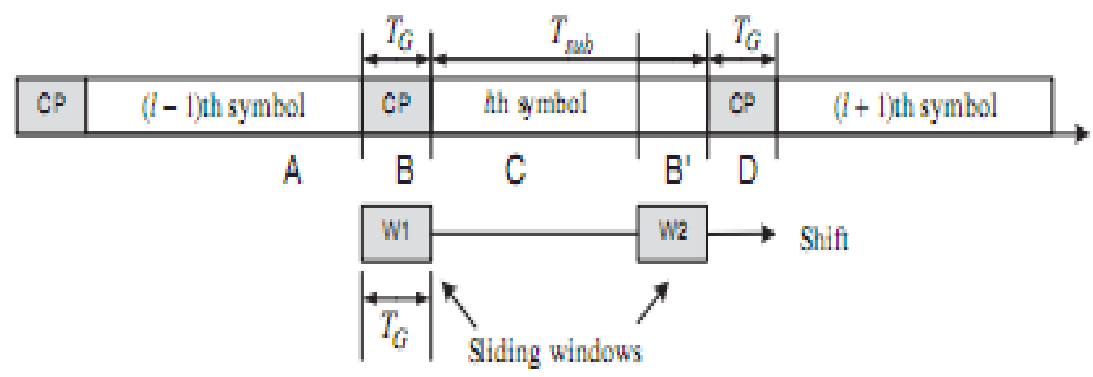

Figure 2: STO estimation technique using double sliding windows.

The estimated STO is done by:

$$
\hat{\delta}=\arg \min _{n}\left\{\sum_{i=\delta}^{N_{G}-1+\delta}\left|y_{l}(n+i)-y_{l}(n+N+i)\right|\right\}
$$

Another technique used in [3]. It consists on minimizing the squared difference between a $\mathrm{N}_{\mathrm{G}}$ sample block (seized in window W1) and conjugate of another NG sample block (seized in window W2).

$$
\hat{\delta}=\arg \min _{n}\left\{\sum_{i=\delta}^{N_{G}-1+\delta}\left(\left|y_{l}(n+i)\right|-\left|y_{l}^{*}(n+N+i)\right|\right)^{2}\right\}
$$

Estimation techniques using training sequences are presented in Figure 3:

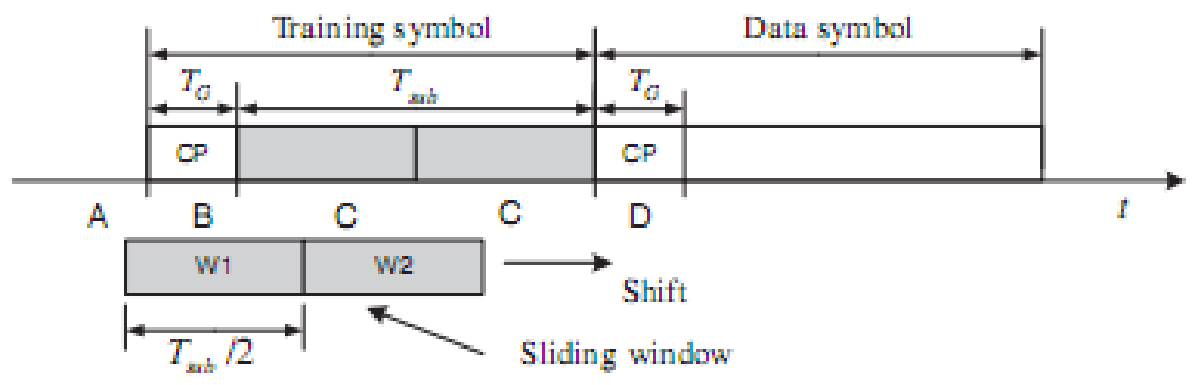

Figure 3: STO estimation using the repetitive training symbol, $($ period $=\mathrm{Tsub} / 2)$. 
In this case, STO is estimated as, [4] and [5]:

$$
\delta=\arg \min _{n}\left\{\sum_{i=\delta}^{N_{G}-1+\delta}\left(\left|y l(n+i)-y_{l}^{*}(n+N / 2+i)\right|\right)^{2}\right\}
$$

In this paper, STO is estimated in frequency domain. As discussed in case II, the received signal suffers from a phase rotation, Figure 4:

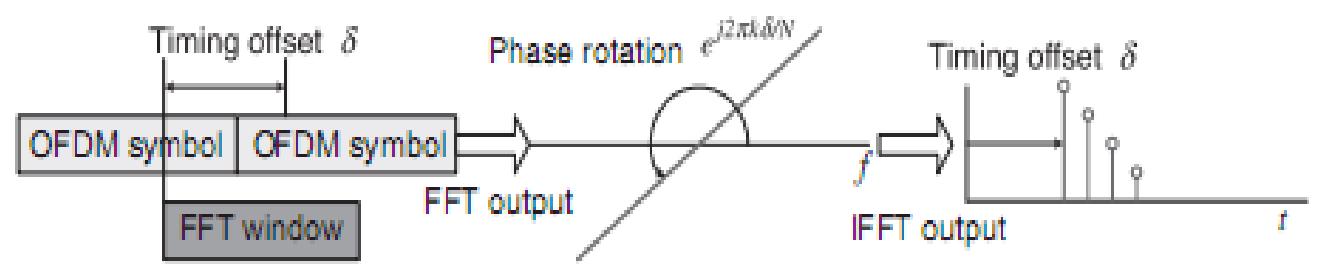

Figure 4 : Effect of STO in frequency domain

STO is estimated as:

Where ${ }^{y_{l}^{X}(n)}$ is defined as:

$$
\delta=\arg \max _{n}\left(y_{l}^{X}(n)\right)
$$

$$
y_{l}^{X}(n)=\operatorname{IFFT}\left\{Y_{l}(k) e^{j 2 \pi \delta k / N} X_{l}^{*}(k)\right\}
$$

\section{Channel Estimation}

Channel estimation techniques for OFDM systems can be divided into two major groups: blind and non-blind. Methods for blind channel estimation exploit the statistical behavior from the received signals and require a lot of data. Therefore, they suffer from serious performance degradation in fast fading channels. Moreover, in the non-blind channel estimation methods, information from precedent channel estimates or a portion of the transmitted signal is present to the receiver to be used for channel estimation. In the data aided channel estimation, known data by the receiver is inserted into the OFDM symbols so the actual channel can be estimated. Two techniques are commonly used: sending known information over one or more OFDM symbols with no data being sent (called channel estimation with training symbols), or sending known information together with the data (called pilots aided channel estimation).

In this paper, we are interested in pilots aided channel estimation, so we will explain in detail this technique with different methods. In the pilots aided channel estimation, the pilots are multiplexed with the data. For time domain estimation, the CIR (Channel Impulse Response) is estimated first. The estimate of the CIR is then passed through a FFT operation to get the channel at each subcarrier for the equalization in frequency domain. For frequency domain estimation, the channel at each pilot is estimated, and then these estimates are interpolated via different methods. Different type of arrangement of pilots is done: block type and comb type. In this paper, we are interested in comb pilot arrangement. 

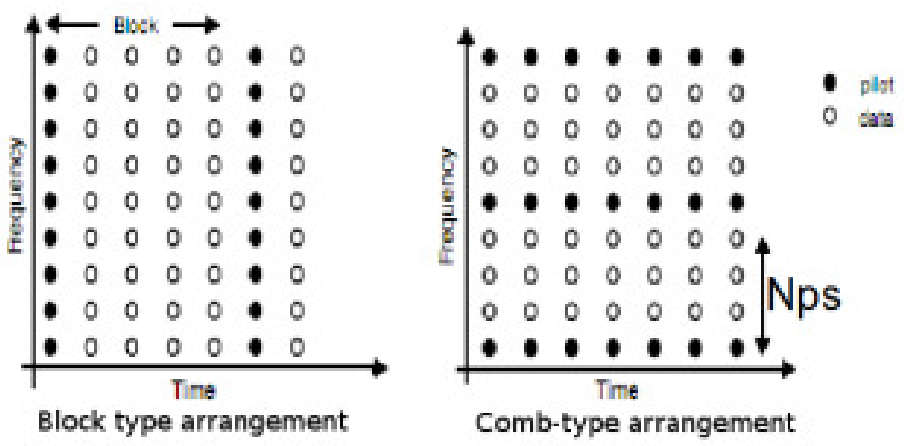

Figure 5: Pilot Arrangement for Channel estimation.

We consider $\mathrm{N}_{\mathrm{p}}$ pilot signals, equi-spaced with spacing $\mathrm{N}_{\mathrm{ps}}$, are inserted into $\mathrm{X}$, where $\mathrm{N}_{\mathrm{ps}}=$ $\mathrm{N} / \mathrm{N}_{\mathrm{p}}$. Receiver knows the locations of pilots $\mathrm{P}=\left[\mathrm{P}_{\mathrm{k}}\right]^{\mathrm{T}}\left(\mathrm{k}=0,1, \ldots, \mathrm{N}_{\mathrm{p}}-1\right)$, the pilot value $\mathrm{X}^{\mathrm{P}}=\left[\mathrm{X}_{\mathrm{k}}^{\mathrm{P}}\right]^{\mathrm{T}}$ and $\mathrm{Y}$ the received signal, [7].

Many techniques used to estimate OFDM channel. The most known are Least Square (LS) and minimum mean square error (MMSE).

\subsection{LS}

Least square method calculates channel response at pilot subcarriers $\mathrm{H}^{\mathrm{P}}$ as follows:

$$
\hat{H}_{L S}^{P}=\left[Y\left(P_{0}\right) / X_{0}^{P}, Y\left(P_{1}\right) / X_{1}^{P}, \ldots, Y\left(P_{N_{P}-1}\right) / X_{N_{P}}^{P}\right]^{T}
$$

The task here is to estimate the channel conditions at the data subcarriers (specified by $\mathrm{H}$ with length $\mathrm{N}$ ), given the LS estimates at pilot subcarriers $\hat{H}_{L S}^{P}$, received signal Y.

\subsection{MMSE}

The MMSE estimator employs the second-order statistics of the channel conditions to minimize the mean-square error.

$$
\hat{H}_{M M S E}^{P}=R_{H A}\left(R_{H H}+\frac{\sigma_{n}^{2}}{\sigma_{z}^{2}} I\right)^{-1} H_{L S}^{P}
$$

Where is the cross-correlation matrix between the true channel vector and temporary channel estimate vector in the frequency domain and $\sigma_{\mathrm{n}}{ }^{2} / \sigma_{\mathrm{x}}{ }^{2}$ is the inverse of the SNR.

To estimate channel in data subcarrier, we need interpolation technique. In the literature, many interpolation techniques are implemented. We explain two of them Linear and second order interpolation [8].

Linear interpolation: The estimated channel response at the data-carrier $\mathrm{k}, \mathrm{mL}<\mathrm{k}<(\mathrm{m}+1) \mathrm{L}$ is given by :

$$
\begin{aligned}
H_{e}(k) & =H_{e}(m L+l) \\
& =\left(H_{p}(m+1)-H_{p}(m)\right) \frac{l}{L}+H_{p}(m)
\end{aligned}
$$


Second Order Interpolation: The estimated channel response is given by:

$$
\begin{aligned}
H_{\mathrm{e}}(k) & =H_{\mathrm{e}}(m L+l) \\
& =c_{1} H_{p}(m-1)+c_{0} H_{p}(m)+c_{-1} H_{p}(m+1)
\end{aligned}
$$

Where $\left\{\begin{array}{l}c_{1}=\frac{\alpha(\alpha-1)}{2} \\ c_{0}=-(\alpha-1)(\alpha+1), \quad \alpha=\frac{1}{N} \\ c_{-1}=\frac{\alpha(\alpha+1)}{2}\end{array}\right.$

Other interpolation techniques are listed in [10] such as Low-pass interpolation, spline cubic interpolation, time domain interpolation...

\section{FRACTIONAL FOURIER TRANSFORM (FRFT)}

The fractional Fourier Transform is a generalisation of the Fourier Transform. The FRFT of a signal $\mathrm{s}(\mathrm{t})$ is defined as follows :

$$
F_{\alpha}(s)=S_{\alpha}(u)=\int_{-\infty}^{+\infty} s(t) K_{\alpha}(t, u) d t .
$$

Where $\mathrm{p}$ is a real number known as FRFT order, $\alpha=\mathrm{p} \pi / 2$ is the angle of FRFT, and $\mathrm{K}_{\alpha}(\mathrm{t}, \mathrm{u})$ is the kernel of FRFT:

$$
= \begin{cases}\sqrt{\frac{1-j \cot (\alpha)}{2 \pi}} \exp \left(j \frac{t^{2}+u^{2}}{2} \cot (\alpha)-j u t c s c(\alpha)\right) & \alpha \neq n \pi \\ \delta(t-u) & \alpha=2 n \pi \\ \delta(t+u) & \alpha+\pi=2 n \pi\end{cases}
$$

Fractional Fourier Transform could be seen as a projection of the signal on an axis which forms an angle $\alpha$ with the time axis: a rotation in the time-frequency plane that generalizes FFT. "The FRFT gives great satisfactions in many signals processing applications such optical communications, signal filtering and also beam forming for fading channels", [9]. Multicarrier modulation that uses traditional Fourier Trans form attempts a frequency windowing of bandwidth. The effect of the time-invariant channel distortions can be compensated for by subchannel-by-subchannel basis single-tap frequency domain equalizers. Consequently, the overall traditional multicarrier system can be seen as an optimal Fourier-domain filter. However, when channel is time-varying, traditional multicarrier system loses optimality since ideal recovery operator is mostly time-variant. This means that it cannot be employed in the conventional Fourier domain and is the reason motivating the use of fractional Fourier transform based technique. 


\section{Proposed Method}

This section deals with the proposed method. A conventional OFDM system is used but Fractional Fourier Transform FRFT block is used instead of classical FFT. We use CAZAC sequences as pilot sequences. The pilot sequences are inserted in combo-type mode. The Timing Offset estimation is done in frequency domain. After STO estimation, compensation is done in time domain. After channel estimation is used in order to best recover the transmitted signal. LS with DFT channel estimator is used with linear interpolation. The DFT-based channel estimation technique has been derived to improve the performance of LS or MMSE channel estimation by eliminating the effect of noise outside the maximum channel delay, [1]. The DFT block diagram is shown in figure 6.

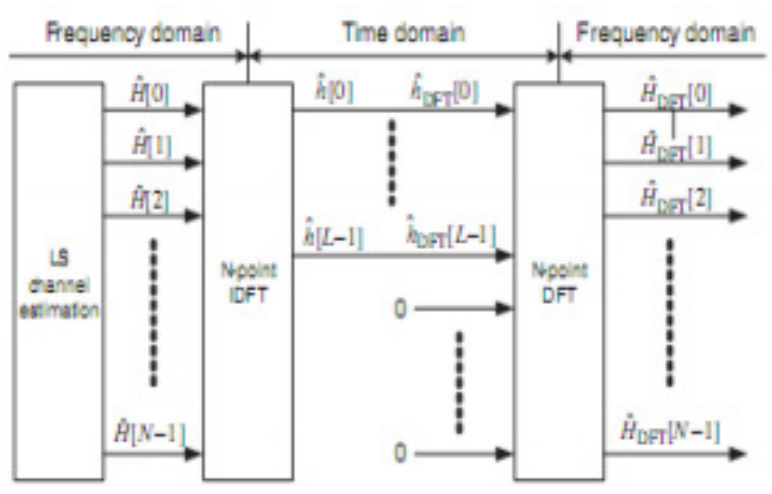

Figure 6 : DFT-based channel estimation.

CAZAC sequences used in this paper are defined as :

$$
X_{p}\left((k-1) * N_{p s}+1\right)=e^{j * \pi *(k-1)^{2} / N_{p}} \quad \text { for } k=1 \ldots N_{p}
$$

Where $\mathrm{N}_{\mathrm{ps}}$ and $\mathrm{N}_{\mathrm{p}}$ are pilot spacing and number of pilotsequences respectively in OFDM symbol. The OFDM basic system of the proposed method is presented in figure 7 .

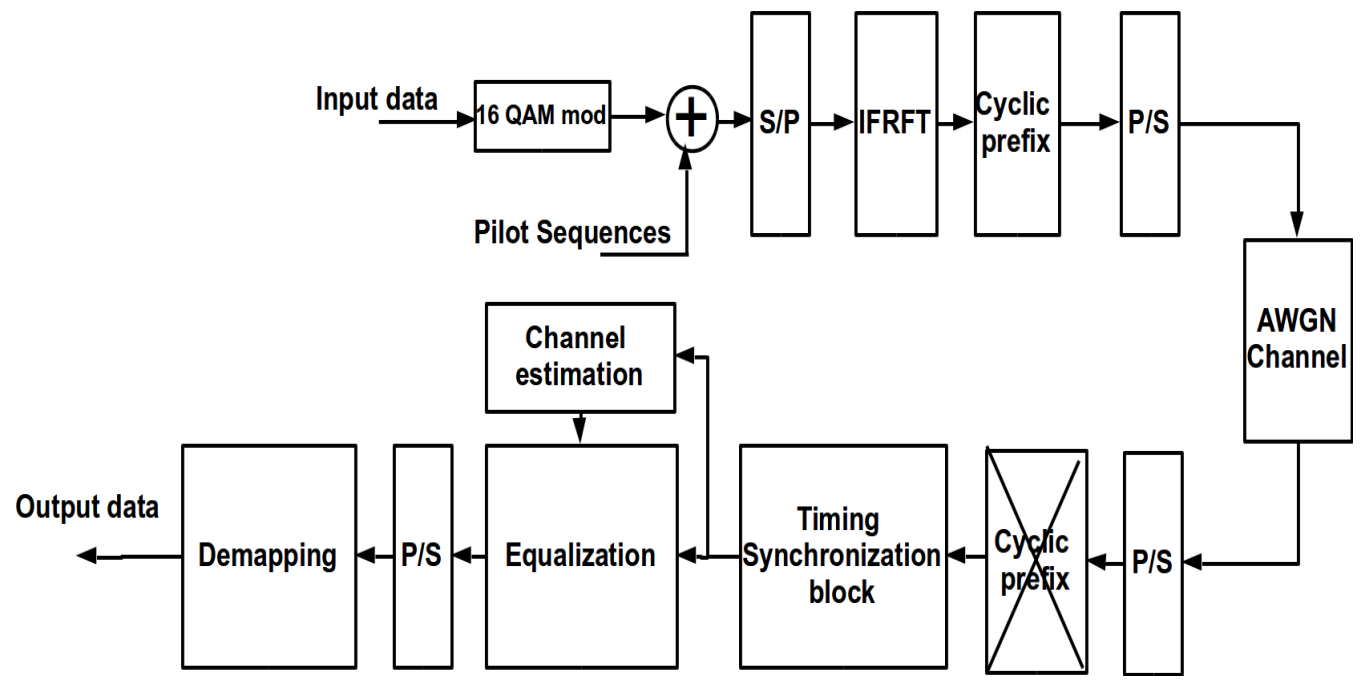

Figure 7: OFDM system of the proposed method. 
Where timing synchronization block is represented in figure 8.

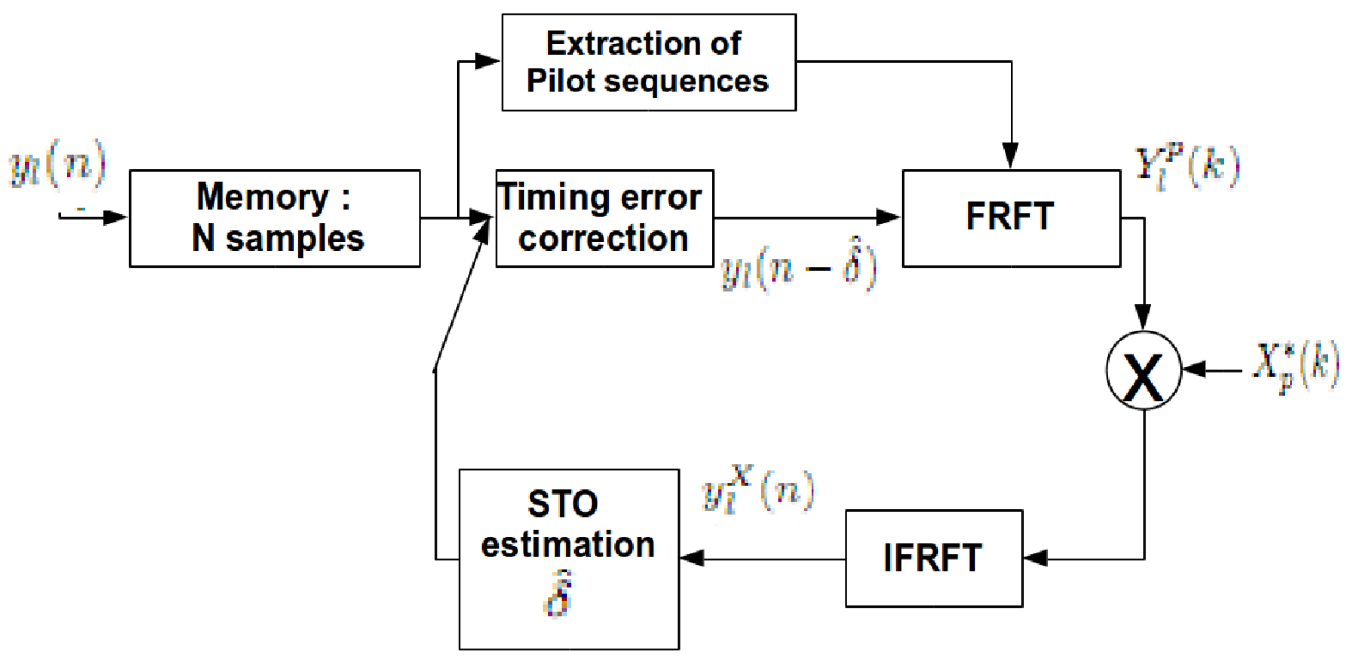

Figure 8: STO synchronization block

\section{Simulations RESUltS AND DisCUSSION}

Parameters of simulation for the proposed method are listed in Table 2.

Table 2: The parameters for simulation.

\begin{tabular}{|l|l|}
\hline Modulation & 16 QAM \\
\hline Number of sub-carrier & 128 \\
\hline Cyclic Prefix Length (Ng) & 32 \\
\hline Pilot sequences & CAZAC sequences \\
\hline Number of Bits per Symbol & 4 \\
\hline Pilot Spacing (Nps) & 4 \\
\hline STO & -3 \\
\hline Number of iteration & 100 \\
\hline Signal to Noise Ratio (SNR) range & $0: 30$ \\
\hline
\end{tabular}

Figure 9 shows the Mean Square Error (MSE) of the Carrier Frequency Offset (STO) of the OFDM system using Fractional Fourier Transform and CAZAC sequences. This figure shows attractive results of the proposed system in terms of MSE by comparison to competitive systems such as Schmidl and Cox[5], Shi and Serpedin [6] methods. Simulation also proves the effect of fractional Fourier transform in comparison with classical Fourier transform. This method is useful in fast varying channel that varies from OFDM symbol to another and does not decrease much the useful throughput in comparison with the methods using training sequences. The proposed method shows attractive results compared to GR method proposed by G. Ren, Y. Chang, and H. Zhang, [11] and better results than the one done by A.P. Rathkanthiwar and A.S.Gandhi, [12]. 
For SNR equal to $15 \mathrm{~dB}$, the proposed method shows a gain ratio in comparison with Shi \& Serpedin method equal to $20 \mathrm{~dB}$.

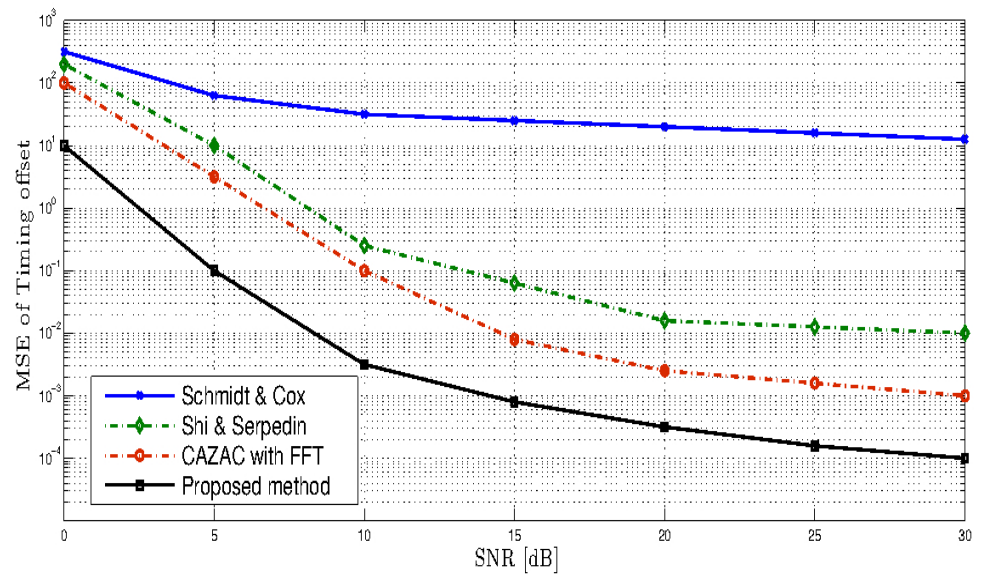

Figure 9: MSE of STO estimation for proposed method compared to known method: Schmidl and Cox, Shi and Serpedin.

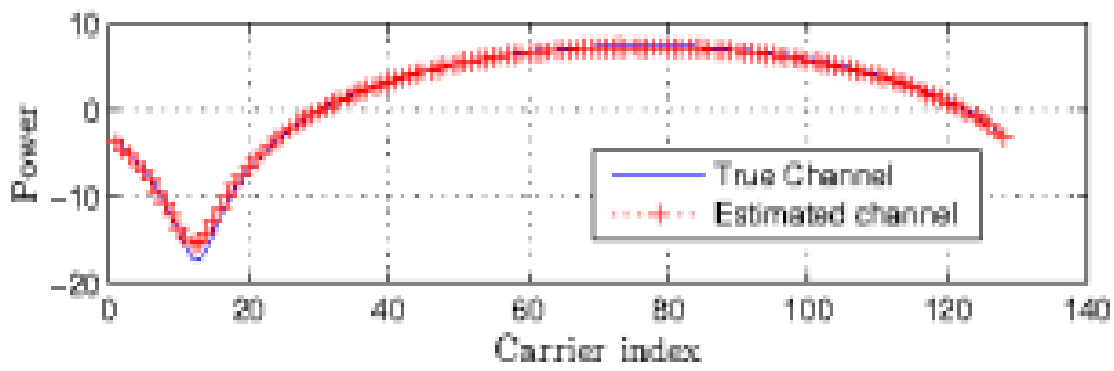

Figure 10: Real channel vs estimated channel for the proposed method for $\mathrm{SNR}=15 \mathrm{~dB}$.

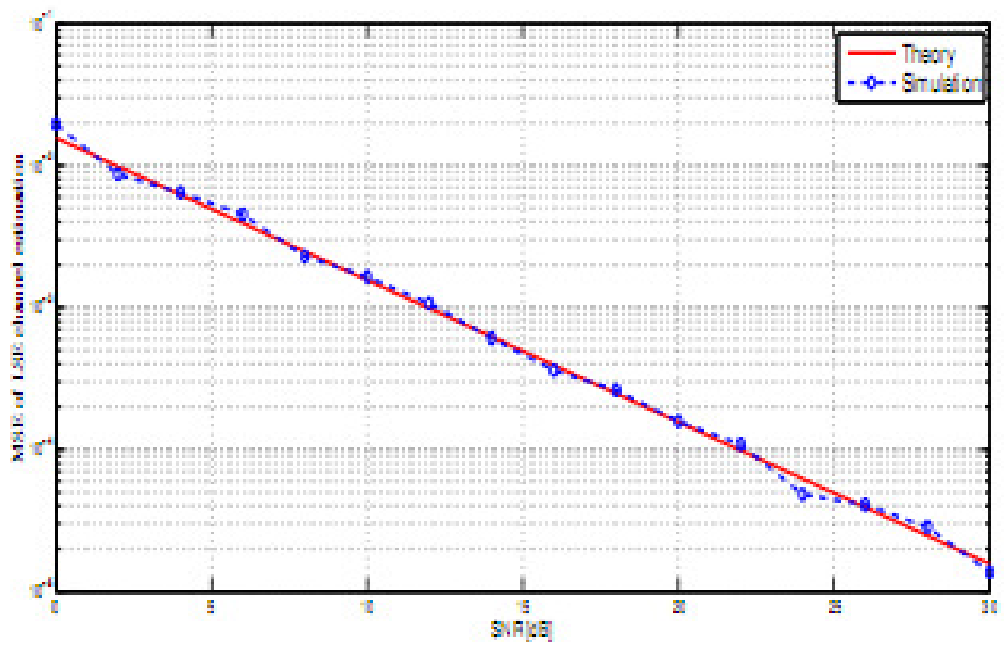

Figure 11: MSE of LSE channel estimation theory and simulation. 
It is shown in figure 10 and figure 11 that our method gives attractive results. The estimated channel is nearly to real channel. The MSE of LSE of the proposed method is almost the theoretical one which gives the proposed method a good candidate for both Symbol Timing Offset and channel estimation.

\section{Conclusions}

This paper proposes new symbol timing offset (STO) and channel estimation method that uses CAZAC sequences as pilot sequences in conjunction with Fractional Fourier Transform. The main design criterion of this method is to exploit the well-known efficiency of both CAZAC sequences and FRFT in reducing MSE of STO of the designed system. The system also gives good results for channel estimation in comparison with the real one. The system we designed shows attractive performance and stands useful for mobile fast varying channels.

\section{REFERENCES}

[1] Cho, Y.S., Kim J., Yang, W.Y. and Kang C. G., "MIMO-OFDM wireless communications with MATLAB”, John Wiley and Sons, Asia, IEEE Press, 2010, pp.153-161.

[2] Tourtier, P.J., Monnier, R., and Lopez, P. "Multicarrier modem for digital HDTV terrestrial broadcasting". Signal Process., 5(5), 379403, 1993.

[3] Speth,M., Classen, F., and Meyr,H. "Frame synchronization of OFDM systems in frequency selective fading channels". IEEE VTC97, pp.18071811, 1997.

[4] T. M. Schmidl and D. C. Cox , "Robust Frequency and Timing Synchronization for OFDM", IEEE Trans. on Commun. , pp.1613 -1621, 1997.

[5] D. C. Schmidl and D. C. Cox , "Low-overhead, low-complexity [burst] synchronization for OFDM", IEEE Int. Conf. on Commun , vol. 3 ,pp.1301 -1306, 1996.

[6] K. Shi and B. E. Serpedin. "Coarse frame and carrier synchronization of OFDM systems: A new metric and comparison", IEEE Trans. Wireless Commun., vol. 3, no. 4, pp. 12711284, Jul. 2004.

[7] Coleri, S., Ergen, M., Puri, A., and Bahai, A.,"'Channel Estimation Techniques Based on Pilot Arrangement in OFDM Systems," IEEE Transactions on Broadcasting, vol.48, pp.223229, Sept. 2002.

[8] Sinem Coleri, Mustafa Ergen,Anuj Puri, Ahmad Bahai, "A Study of Channel Estimation in OFDM Systems", IEEE 56th Vehicular technology conference.

[9] Massimiliano (Max) Martone , "A Multicarrier System Based on the Fractional Fourier Transform for Time-Frequency-Selective," IEEETransactions on Communications, VOL. 49, NO. 6, June 2001.

[10] Yushi Shen and Ed Martinez,"Channel Estimation in OFDM Systems", Freescale Semiconductor, Inc., 2006.

[11] G. Ren, Y. Chang, and H. Zhang, "Synchronization method based on a new constant envelop preamble for OFDM systems,"IEEE Trans.Broadcast.,vol. 51, no. 1, pp. 139-143, Mar. 2005.

[12] A.P. Rathkanthiwar and A.S. Gandhi, "A new timing metric for timing error estimation in OFDM", conference on Wireless Communications, Vehicular Technology, Information Theory and AerospaceElectronics Systems (VITAE), 2013. 
International Journal of Computer Networks \& Communications (IJCNC) Vol.8, No.2, March 2016

\section{Authors}

Cherif Rezgui is graduate as telecommunication Engineer from higher school of - communication in 2004, his master degree in 2011 from national school of engineers of Tunis $\mathrm{He}$ is actually working on $\mathrm{Ph}$.
estimation and synchronization.

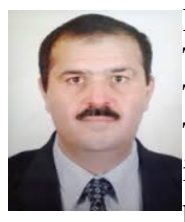

Khaled Grayaa is a Professor at National School of Advanced Sciences and Technologies (ENSTAB). He was Director of the National Higher Engineering School of Tunis from 2011 to 2015. He received his Phd in 2000 and HDR in 2007 in Telecommunication from the National Engineering School of Tunis. His interests include microwave circuits modelling, wireless communications and smart grid. He has published 25 articles in refereed conference lectures and 15 journal articles. 